\title{
Manageability of Acute Severe Heart Failure Complicated With Left Ventricular Thrombosis During Therapy for Breast Cancer
}

\author{
Sebastian Szmit, ${ }^{1,4}$ MD, Marcin Kurzyna, ${ }^{2}$ MD, Renata GŁówczyńsKa, ${ }^{1}$ MD, Marcin Grabowski, ${ }^{1}$ MD, \\ Jarosław Kober, ${ }^{2}$ MD, Justyna Czerniawska, ${ }^{3}$ MD, Krzysztof J FilipiaK, ${ }^{1}$ MD, Grzegorz Opolski, ${ }^{1}$ MD, \\ and Cezary SzCZYLiK, ${ }^{4} \mathrm{MD}$
}

\begin{abstract}
SUMMARY
Trastuzumab-related cardiac dysfunction may be manageable and completely reversible with suitable cardiac medication, allowing optimal breast cancer treatment to continue. We present the case of a 42 -year-old woman who developed severe systolic left ventricular failure with impaired contractility of the right ventricle, pulmonary hypertension, and clots in the left ventricular cavity during adjuvant treatment for breast cancer. The patient was initially diagnosed with early breast cancer and underwent surgery on her left breast. She received 6 cycles of anthracycline chemotherapy followed by radiation therapy in the left breast area, then 5 cycles of trastuzumab. After the fifth cycle of trastuzumab, she experienced dyspnoea and leg edema. Fluid was detected in the pleural cavities but no lung metastases were identified. Echocardiography was performed, revealing a severely reduced left ventricular ejection fraction $(10 \%)$ with impaired contractility of the right ventricle and pulmonary hypertension. Standard medication for heart failure resulted in complete recovery of normal systolic and diastolic function of the left and right ventricles. The combination of low molecular weight heparin and acetylsalicylic acid completely resolved the thrombotic complications. The patient regained her full range of social, occupational, and family activities. This case study is the first to demonstrate the manageability and reversibility of trastuzumab-related cardiac complications in a patient who had developed severe heart failure complicated with left ventricular thrombosis during sequential anthracycline and trastuzumab therapy for breast cancer. The findings contradict other opinions that trastuzumab-related acute heart failure is analogous to stunning or hibernation and recovers without specific cardiac treatment. (Int Heart J 2010; 51: 141-145)
\end{abstract}

Key words: Acute heart failure, Trastuzumab, Anthracycline, Early breast cancer, Ventricular thrombosis

$\mathrm{F}$ our large, multicentre, randomised, pivotal, adjuvant trastuzumab (Herceptin ${ }^{\circledR}$; F Hoffmann-La Roche Ltd, Basel, Switzerland) trials [HERceptin Adjuvant (HERA), National Surgical Adjuvant Breast and Bowel Project B-31, North Central Cancer Treatment Group N9831 and Breast Cancer International Research Group (BCIRG) 006] have demonstrated that adjuvant trastuzumab treatment after standard adjuvant chemotherapy provides significant clinical benefits for women with human epidermal growth factor receptor 2 (HER2)-positive breast cancer, compared with chemotherapy alone. ${ }^{1-3)}$ As a result of these trials, trastuzumab is now recognised as the standard of care in women with HER2-positive breast cancer and has been administered to more than half a million patients to date.

However, the incidence of cardiac side effects, including congestive heart failure (CHF), in early pivotal trastuzumab trials was higher than expected, particularly when trastuzumab was given concurrently with anthracyclines. ${ }^{3-6)}$ Cardiac safety during trastuzumab-anthracycline regimens in the adjuvant setting has, therefore, become a priority.

Trastuzumab-related cardiac dysfunction - Type II chemotherapy-related cardiac dysfunction (CRCD) - is recognised as a distinct entity from that associated with anthracyclines (Type I CRCD). ${ }^{7)}$ Type II CRCD is not dose-related, although it may improve spontaneously with the continuation or discontinuation of trastuzumab, and is largely reversible. ${ }^{7.8)}$ Conversely, anthracyclines are known to cause cumulative, permanent, and irreversible myocardial damage. ${ }^{9,10)}$

The risk of cardiac dysfunction must, therefore, be balanced with the expected benefit of treatment for every patient undergoing breast cancer therapy. We present the first case study demonstrating that severe heart dysfunction may be successfully managed with the correct standard of cardiac medication in a patient who has received optimal breast cancer treatment.

\section{Case Report}

In May 2006, following ultrasound examination, a solid tumour $(13 \times 10 \times 8 \mathrm{~mm})$ with a small calcification area was identified within the left breast of a 42-year-old woman. Six months later, tumourectomy and histopatho-

From the ${ }^{1}$ First Department of Cardiology, Medical University of Warsaw, Departments of ${ }^{2}$ Chest Medicine and ${ }^{3}$ Lung Diseases, National Tuberculosis and Lung Diseases Research Institute, and ${ }^{4}$ Department of Oncology, Military Institute of Health, Warsaw, Poland.

Address for correspondence: Sebastian Szmit, MD, First Department of Cardiology, Medical University of Warsaw, Banacha Street 1a, 02-097 Warsaw, Poland. Received for publication October 22, 2009

Revised and accepted December 21, 2009. 


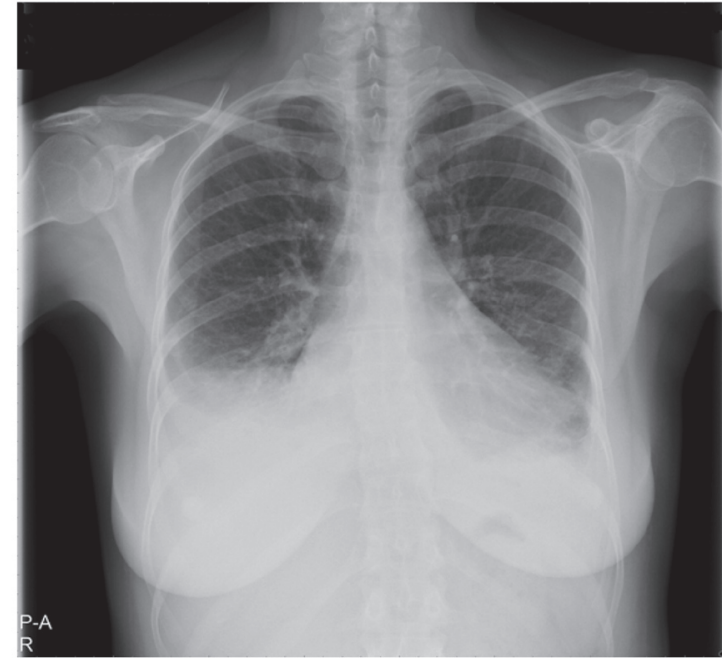

Figure 1. Chest X-ray revealed the presence of fluid within both pleural cavities in the present female case with acute heart failure due to breast cancer treatment.

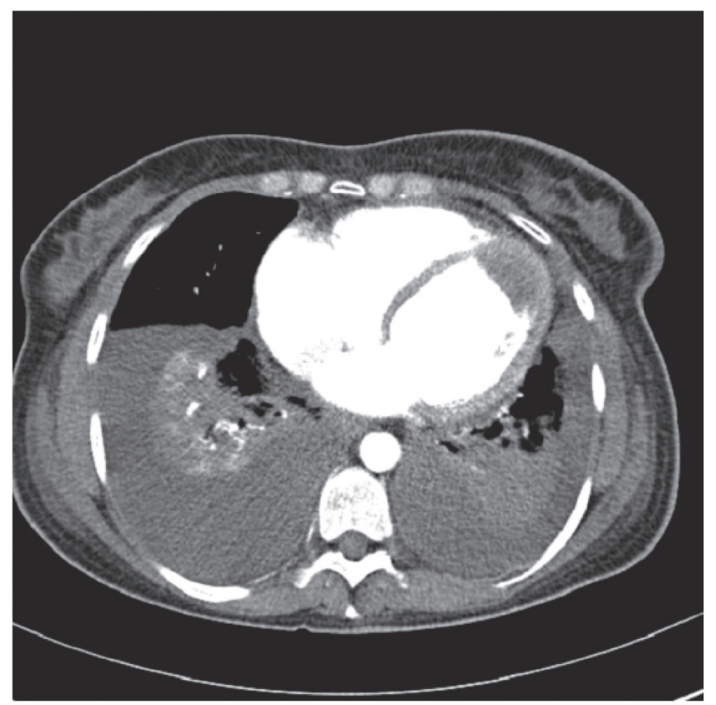

Figure 2. Computed tomography identified a clot in the left ventricular cavity.

logical examination were performed. Although cancer metastases were not detected in any of the 11 lymph nodes examined, the solid tumour was diagnosed as invasive lobular breast carcinoma G1 (pT1c) with a lobular carcinoma structure (classical variant) and a maximum diameter of 1.2 $\mathrm{cm}$. Molecular tests revealed that the tumour was oestrogenreceptor and progesterone-receptor negative. Immunohistochemistry (IHC), using the Dako HercepTest ${ }^{\mathrm{TM}}$, identified the tumour as being HER2 positive (IHC $3+$ ). The patient received 6 cycles of $60 \mathrm{mg} / \mathrm{m}^{2}$ doxorubicin in combination with $900 \mathrm{mg}$ cyclophosphamide following surgery. Then, 5 months postsurgery, she underwent radiation therapy for

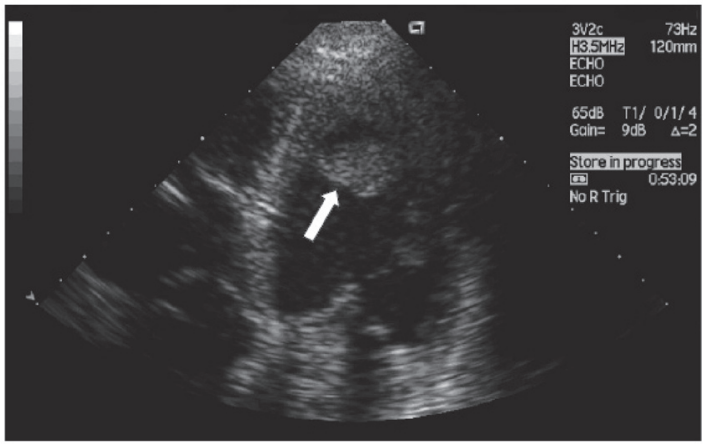

Figure 3. Echocardiograms showing a clot in the left ventricle, as indicated by a white arrow.

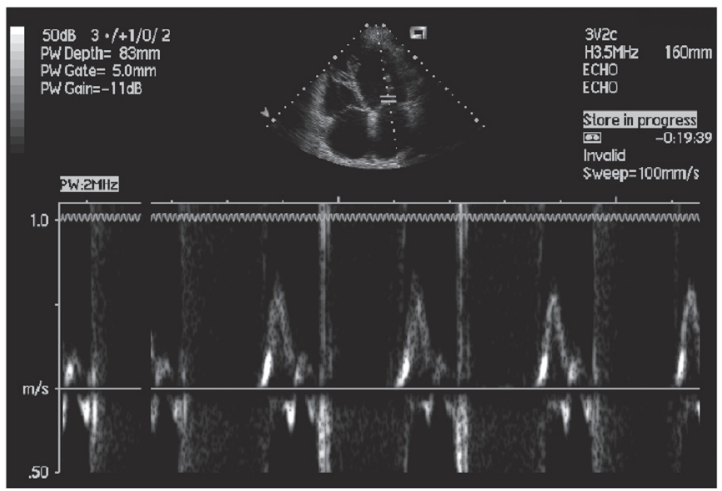

Figure 4. Echocardiogram showing restrictive mitral flow.

2 months according to an individual therapeutic plan: 5000 cGy/t (200 cGy/t in 25 fractions) to the left breast area and $1400 \mathrm{cGy} / \mathrm{t}$ (200 cGy/t in 7 fractions) to the site of the left breast from where the tumour was excised. At this point, the patient had no risk factors for atherosclerosis, normal electrocardiography, normal echocardiographical findings, and no clinical symptoms of heart disease. Therefore, no further cardiac evaluations were performed at this time.

Starting 8 months postsurgery, the patient received trastuzumab at a loading dose of $8 \mathrm{mg} / \mathrm{kg}$ and then a maintenance dose of $6 \mathrm{mg} / \mathrm{kg}$ every 3 weeks. After 3 months, the patient developed dyspnea (New York Heart Association [NYHA] class II) and a respiratory infection was diagnosed, which the oncologist treated with antibiotics. During the following 10 days, the patient developed exercise and resting dyspnea (NYHA class IV) and leg edema, and subsequently was admitted to hospital with severe heart failure. A chest X-ray revealed the presence of fluid within both pleural cavities (Figure 1). The patient had no atherosclerosis risk factors, there was no family history of cardiomyopathy, and she did not present with any arrhythmias or atrio-ventricular disturbances, which excluded a diagnosis of tachyarrhythmia-induced cardiomyopathy. A 64-row computed tomography scan was performed. No lung metastases were observed and her coronary arteries 
showed no sign of atherosclerotic changes or congenital disorders, but the scan revealed a possible metastasis within the left ventricular cavity (Figure 2). Magnetic resonance imaging of the heart excluded metastases and changes typical of myocarditis. Since this area was covered by a clot, echocardiography was performed for verification. Severe contractility disorders of the left ventricle were observed: a

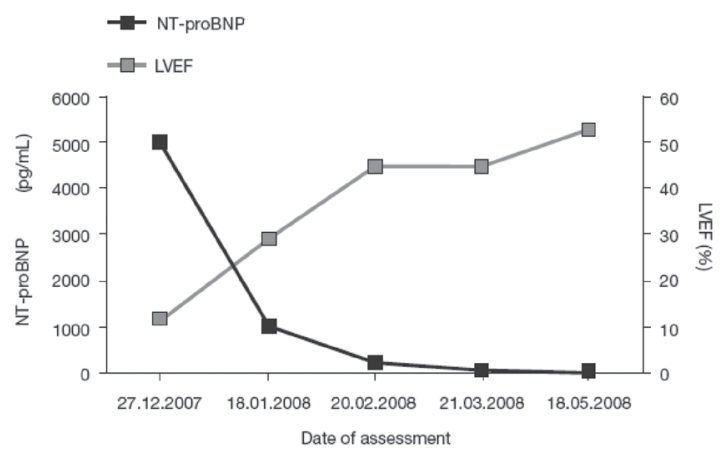

Figure 5. Chart showing the association of NTproBNP (BNP = B-type natriuretic peptide) decrease with LVEF improvement during followup. minimum left ventricular ejection fraction (LVEF) of $10 \%$, a clot within the left ventricle (Figure 3 ) and restrictive mitral flow (Figure 4) with accompanying hypokinesia of the right ventricle and symptoms of pulmonary hypertension. Details of all cardiovascular complications are provided in Table.

Consequently, a continuous infusion of unfractionated heparin was given to induce anticoagulation. During this infusion, the thrombus in the left ventricle enlarged and a second lesion was detected. However, following the introduction of low molecular weight heparin (enoxaparine 100 $\mathrm{mg}$ /day subcutaneously) combined with acetylsalicylic acid (75 mg once daily), complete regression of these severe lesions in the left ventricle was observed. Congestive heart failure was treated by the extraction of $550 \mathrm{~mL}$ of aseptic, clear fluid, with a specific gravity of $1020 \mathrm{~g} / \mathrm{cm}^{3}$, from the pleural cavities. Subsequently, the optimum medication for heart failure was introduced: enalapril ( $5 \mathrm{mg} / \mathrm{day})$, carvedilol (25 mg/day), torasemide ( $5 \mathrm{mg} /$ day $)$, spironolactone $(12.5 \mathrm{mg} /$ day $)$, and digoxin $(125 \mu \mathrm{g} /$ day $)$. The optimum dose of some medications (ie, carvedilol, enalapril) was reached by gradually increasing the dose levels. Trastuzumab treatment was not continued.

As a result of the treatment, a significant improvement was observed in the patient's clinical condition, with

Table. Main Clinical and Laboratory Findings

\begin{tabular}{|c|c|c|}
\hline Examination date & Echocardiography findings & Other \\
\hline 18 December 2006 (baseline) & Normal global and segmental contractility & $\begin{array}{l}\text { No symptoms } \\
\text { Normal ECG }\end{array}$ \\
\hline 14 December 2007 & $\begin{array}{l}\text { Generalized severe hypokinesia } \\
\text { Significant hypokinesia of the right ventricle } \\
\text { Signs of pulmonary hypertension } \\
\text { Large additional mass in the left ventricle }(4.5 \times 2 \times 1.5 \mathrm{~cm}) \text {, } \\
\text { most likely a clot }\end{array}$ & $\begin{array}{l}\text { Resting dyspnea (NYHA IV) } \\
\text { Sinus tachycardia ( } 115 \mathrm{bpm} \text { ) } \\
\text { An abnormal Q-wave (or QS complex) in leads } \\
\mathrm{V}_{1}-\mathrm{V}_{2} \\
\text { Weak progression of R-waves in } \mathrm{V}_{3}-\mathrm{V}_{4} \\
\text { Low voltage of QRS complexes in the limb } \\
\text { leads and a negative T-wave in } \mathrm{V}_{5}-\mathrm{V}_{6} \text {. }\end{array}$ \\
\hline 27 December 2007 & $\begin{array}{l}\text { Very significantly impaired persistent contractility of the left } \\
\text { ventricle, nearly complete akinesia } \\
\text { Lesion in the left ventricle further enlarged } \\
\text { LVEF } 10 \% \\
\text { An additional mobile structure with a rather thin pedicle in the } \\
\text { apical region }(1.5 \times 1.5 \mathrm{~cm}) \\
\text { Immobile inferior vena cava on respiration, not dilated }(19 \mathrm{~mm}) \\
\text { Borderline systolic pressure in the pulmonary artery }\end{array}$ & $\begin{array}{l}\text { Resting dyspnea (NYHA IV) } \\
\text { NT-proBNP } 5032 \mathrm{pmol} / \mathrm{L} \\
\text { Troponin } 0.0 \mathrm{ng} / \mathrm{Ml} \\
\text { ECG as above }\end{array}$ \\
\hline 6 January 2008 & $\begin{array}{l}\text { Lesions in the left ventricle almost completely disappeared } \\
\text { No changes in other parameters }\end{array}$ & $\begin{array}{l}\text { Resting dyspnea (NYHA IV) } \\
\text { ECG as above }\end{array}$ \\
\hline 16 January 2008 & $\begin{array}{l}\text { Akinetic interventricular septum } \\
\text { LVEF } 29 \% \\
\text { Restrictive type of inflow to the left ventricle } \\
\text { Improved function of the right ventricle (TAPSE } 10 \mathrm{~mm} \text { ) }\end{array}$ & $\begin{array}{l}\text { Exercise dyspnea (NYHA II) } \\
\text { NT-proBNP } 1070 \mathrm{pmol} / \mathrm{L} \\
\text { ECG as above }\end{array}$ \\
\hline 20 February 2008 & $\begin{array}{l}\text { Hypokinesia of the interventricular septum in the apical and } \\
\text { medium segments } \\
\text { Normal diastolic function of the left ventricle } \\
\text { LVEF } 45 \% \\
\text { Maintained function of the right ventricle (TAPSE } 17 \mathrm{~mm} \text { ) }\end{array}$ & $\begin{array}{l}\text { Exercise dyspnea (NYHA I) } \\
\text { Normalization of ECG }\end{array}$ \\
\hline 21 March 2008 & $\begin{array}{l}\text { No contractility disorders of the left ventricle } \\
\text { LVEF } 54 \% \\
\text { Maintained function of the right ventricle (TAPSE } 21 \mathrm{~mm} \text { ) }\end{array}$ & $\begin{array}{l}\text { No symptoms. Normal ECG } \\
\text { NT-proBNP } 61 \mathrm{pmol} / \mathrm{L}\end{array}$ \\
\hline 18 May 2008 & LVEF $53 \%$ & $\begin{array}{l}\text { No symptoms. Normal ECG } \\
\text { NT-proBNP } 43 \mathrm{pmol} / \mathrm{L}\end{array}$ \\
\hline
\end{tabular}


notable regression of dyspnea (which improved to NYHA class I) and edema. Follow-up echocardiography showed a significant improvement in the systolic and diastolic function of the left ventricle and improved function of the right ventricle. Complete recovery was recorded 4 months after the onset of cardiac dysfunction. The patient had regained full mobility without dyspnea and resumed a fully active family life, returning to work 1.5 months later. No further treatment with trastuzumab was required, as control computed tomography scans showed no evidence of neoplastic disease. The patient remained free of cancer progression up to the last clinic visit on 5 October 2009. However, treatment for heart failure is still continuing. Each reduction of these medications causes asymptomatic ejection fraction decreases of $\leq 45 \%$ (more than 1-year observation).

\section{Discussion}

This case study documents a severe form of heart failure which had developed during adjuvant breast cancer therapy and describes how these cardiac complications were resolved over a short period of time using optimal pharmacotherapy of heart failure.

It documents that trastuzumab-related acute severe heart failure may not be analogous to heart stunning or hibernation and does not recover without specific cardiac treatment, such as angiotensin-converting enzyme inhibitor, beta-blocker, digoxin, or diuretic (spironolactone and torasemide) administration. ${ }^{7,8)}$ The patient experienced the progression of severe symptoms of heart failure although trastuzumab was stopped. The long-term follow-up shows that each reduction of the optimal dose of cardiac medications causes LVEF decreases of $\leq 45 \%$ (more than 1-year observation).

Results from the HERA trial demonstrated that 1 year of weekly trastuzumab, after standard adjuvant chemotherapy, significantly improved overall survival (hazard ratio $0.66 ; P=0.0115$ ) and progression-free survival (hazard ratio $0.64 ; P<0.0001$ ) for patients with HER2-positive early breast cancer. ${ }^{11)}$ The incidence of cardiac dysfunction was very low in this trial: $0.60 \%$ of patients had severe CHF, $2.15 \%$ had symptomatic CHF, and $3.04 \%$ had significant LVEF decline. ${ }^{12)}$ No cardiac deaths occurred in the trastuzumab group.

It should be emphasized that higher cumulative doses of doxorubicin and epirubicin had been received by patients who developed cardiac dysfunction in the trastuzumab group compared with the observation-alone group: 287 $\mathrm{mg} / \mathrm{m}^{2}$ versus $257 \mathrm{mg} / \mathrm{m}^{2}$ doxorubicin, and $480 \mathrm{mg} / \mathrm{m}^{2}$ versus $422 \mathrm{~g} / \mathrm{m}^{2}$ epirubicin, respectively. ${ }^{12)}$ In addition, these patients had lower baseline LVEF values and a higher body mass index (BMI) than the observation-alone patients who experienced cardiac dysfunction. ${ }^{12)}$ However, in the majority of patients, cardiac dysfunction was reversible and LVEF returned to normal values within 6 months after stopping trastuzumab.

In this context, the case study presented here is of significant clinical interest, as the patient was young, had a low BMI, a good LVEF and no cardiovascular risk factors. However, she developed acute severe cardiac dysfunction after at least 6 cycles of doxorubicin $\left(60 \mathrm{mg} / \mathrm{m}^{2}\right)$ given sequentially with trastuzumab.

In the pivotal adjuvant N9831 trial, patients with a reduction in LVEF following anthracycline therapy were found to have a significantly higher risk of developing CHF during trastuzumab therapy than those who had not received anthracyclines. ${ }^{13)}$ Therefore, it appears that in this patient the anthracycline treatment resulted in the first irreversible changes in the heart muscle, though these changes were not associated with any clinical symptoms or changes in echocardiography. Possibly, further treatment with trastuzumab blocked the cardioprotective HER2 pathway, ${ }^{14)}$ causing the observed systolic dysfunction and progression to symptomatic heart failure. If this patient had received a cumulative dose of doxorubicin of only $240 \mathrm{mg} / \mathrm{m}^{2}$ the risk of severe heart failure associated with continued trastuzumab would have been significantly lower. While genetic predisposition is obviously a factor, the total dose of anthracycline seems to be fundamental to developing cardiac dysfunction during breast cancer therapy.

Trastuzumab given concurrently with anthracycline may be more effective than sequential trastuzumab-based regimens, but it has not yet been tested in the adjuvant setting. Therapeutic regimens without anthracyclines are being investigated for adjuvant breast cancer: the triplecombination regimen of docetaxel/carboplatin/trastuzumab in the BCIRG 006 trial $^{3)}$ and the combination of liposomal or pegylated anthracyclines with trastuzumab in the Breast cancer Adjuvant Caelyx Herceptin trial. ${ }^{15)}$

Current guidelines for the management of cardiac dysfunction during adjuvant trastuzumab therapy recommend stringent selection criteria for patients, identification of any potential cardiac risk factor before treatment starts, close cardiac monitoring throughout treatment, identification of cases of early asymptomatic LVEF decline before the onset of symptomatic heart failure, and a close working partnership between oncologists and cardiologists to manage cardiac dysfunction. ${ }^{16,17)}$

Despite patients with a good baseline ejection fraction of $\geq 50 \%$ being enrolled in all trastuzumab-based clinical trials, systolic dysfunction was reported in $>20 \%$ of patients and severe cardiac failure in $<4 \%$. $^{1,3,13,18)}$ The question, therefore, remains as to whether it is ethical to withhold breast cancer treatment (including trastuzumab) in patients with mild baseline heart failure, especially in the adjuvant setting where the ultimate goal is to cure the patient. If it is possible to administer efficient cardiac treatment for such a severe form of iatrogenic heart failure as that described here, it is controversial to deprive patients, with either a moderate cardiovascular risk or mild baseline cardiac failure, of breast cancer treatment. In this situation, optimal cardiac treatment enabled optimal breast cancer therapy.

Another important question is how to provide effective anticoagulation in cancer patients. At admission the patient was experiencing perimural thrombus in the left ventricle. Intravenous infusion of unfractionated heparin did not resolve this, but the combination of low molecular weight heparin and aspirin (ASA) allowed for complete resolution of the thrombosis. This shows a synergistic effect of low molecular weight heparin, which seems to be the most ef- 
fective anticoagulant in cancer related venous thromboembolism, and ASA, which is a potent antiplatelet drug of proven efficacy in arterial thrombosis. The anticoagulant treatment in this case was crucial.

Conclusions: Despite having no classical cardiovascular risk factors prior to breast cancer therapy, the patient described here developed a very severe form of iatrogenic heart failure and thrombosis in the left ventricular cavity, following breast cancer treatment. Standard medication for heart failure (ie, angiotensin-converting enzyme inhibitor, beta-blocker, digoxin, diuretics (spironolactone and torasemide)) and medication for thrombosis complications (ie, low molecular weight heparin and acetylsalicylic acid) ensured complete reversibility of these symptoms within a relatively short time period, allowing the patient to receive the optimal treatment for her breast cancer.

\section{REFERENCES}

1. Piccart-Gebhart MJ, Procter M, Leyland-Jones B, et al. Trastuzumab after adjuvant chemotherapy in HER2-positive breast cancer. N Engl J Med 2005; 353: 1659-72.

2. Romond EH, Perez EA, Bryant J, et al. Trastuzumab plus adjuvant chemotherapy for operable HER2-positive breast cancer. N Engl J Med 2005; 353: 1673-84.

3. Slamon D, Eiermann W, Robert N, et al. BCIRG 006: 2nd interim analysis phase III randomized trial comparing doxorubicin and cyclophosphamide followed by docetaxel $(A C \circledR T)$ with doxorubicin and cyclophosphamide followed by docetaxel and trastuzumab $(\mathrm{AC} \otimes \mathrm{TH})$ with docetaxel, carboplatin and trastuzumab (TCH) in Her2neu positive early breast cancer patients. http://www.abstracts2view.com/sabcs06/view. php?nu=SABCS06L_78.

4. Baselga J. Herceptin alone or in combination with chemotherapy in the treatment of HER2-positive metastatic breast cancer: pivotal trials. Oncology 2001; 61: 14-21. (Review)

5. Seidman A, Hudis C, Pierri MK, et al. Cardiac dysfunction in the trastuzumab clinical trials experience. J Clin Oncol 2002; 20: $1215-21$

6. Slamon DJ, Leyland-Jones B, Shak S, et al. Use of chemotherapy plus a monoclonal antibody against HER2 for metastatic breast cancer that overexpresses HER2. N Engl J Med 2001;
344: 783-92.

7. Ewer MS, Lippman SM. Type II chemotherapy-related cardiac dysfunction: time to recognize a new entity. J Clin Oncol 2005; 23: $2900-2$

8. Ewer MS, Vooletich MT, Durand JB, et al. Reversibility of trastuzumab-related cardiotoxicity: new insights based on clinical course and response to medical treatment. J Clin Oncol 2005; 23: 7820-6.

9. Lefrak EA, Pitha J, Rosenheim S, Gottlieb JA. A clinicopathologic analysis of adriamycin cardiotoxicity. Cancer 1973; 32: 302-14

10. Von Hoff DD, Layard MW, Basa P, et al. Risk factors for doxorubicin-induced congestive heart failure. Ann Intern Med 1979; 91: 710-7.

11. Smith I, Procter M, Gelber RD, et al. 2-year follow-up of trastuzumab after adjuvant chemotherapy in HER2-positive breast cancer: a randomised controlled trial. Lancet 2007; 369: 29-36.

12. Suter TM, Procter M, van Veldhuisen DJ, et al. Trastuzumabassociated cardiac adverse effects in the herceptin adjuvant trial. J Clin Oncol 2007; 25: 3859-65.

13. Perez EA, Suman VJ, Davidson NE, et al. Cardiac safety analysis of doxorubicin and cyclophosphamide followed by paclitaxel with or without trastuzumab in the North Central Cancer Treatment Group N9831 adjuvant breast cancer trial. J Clin Oncol 2008; 26: 1231-8.

14. Lemmens K, Doggen K, De Keulenaer GW. Role of neuregulin-1/ErbB signaling in cardiovascular physiology and disease: implications for therapy of heart failure. Circulation 2007; 116: 954-60. (Review)

15. Rayson D, Richel D, Chia S, Jackisch C, van der Vegt S, Suter T. Anthracycline-trastuzumab regimens for HER2/neu-overexpressing breast cancer: current experience and future strategies. Ann Oncol 2008; 19: 1530-9.

16. Jones AL, Verrill M, Barrett-Lee P, et al. Development and implementation of new UK National Cancer Research Institute (NCRI) guidelines for preventing and managing cardiac events with trastuzumab. Ann Oncol 2008; 19: viii85.

17. Mackey JR, Clemons M, Côté MA, et al. Cardiac management during adjuvant trastuzumab therapy: recommendations of the Canadian Trastuzumab Working Group. Curr Oncol 2008; 15: 24-35.

18. Perez EA, Romond EH, Suman VJ, et al. Updated results of the combined analysis of NCCTG N9831 and NSABP B-31 adjuvant chemotherapy with/without trastuzumab in patients with HER2-positive breast cancer. J Clin Oncol 2007; 25: 512. 